> Les dossiers de santé électroniques hospitaliers contribuent à l'amélioration de la qualité des soins en permettant une meilleure gestion des informations cliniques. Les bases de données numériques ainsi constituées facilitent l'échange des informations de santé avec les prestataires de soins et optimisent la coordination multidisciplinaire pour de meilleurs résultats thérapeutiques. Le projet européen EHR4CR (electronic health records for clinical research) a développé une plateforme pilote innovante permettant de réutiliser ces données numériques pour la recherche clinique. En améliorant et en accélérant les procédures de recherche clinique, cette approche permet d'envisager la réalisation d'études cliniques de manière plus efficiente, plus rapide et plus économique. <

Les dossiers de santé électroniques (DSE) permettent de réunir, suivre et partager d'innombrables données cliniques numérisées [1], contribuant à la fois à la coordination des soins pluridisciplinaires et à la qualité des soins [2, 3]. Dès lors que la prise en charge optimale d'un patient requiert la coordination étroite de services prodigués par plusieurs prestataires de soins, la portabilité, l'interopérabilité et l'accès aux données cliniques se révèlent très utiles pour la qualité du suivi et l'atteinte des objectifs thérapeutiques [3, 4]. La médecine personnalisée visant une meilleure prise en compte des éléments cliniques spécifiques est également avide de données, avec, entre autres, celles contenues dans les DSE [5].

L'émergence des nouvelles technologies de l'information, incluant l'avènement des objets connectés et les nombreuses applications d'auto-gestion de la santé, permettent de plus en plus aux patients de saisir leurs données sous forme numérique, et d'en autoriser l'accès aux professionnels de santé. Ces avancées contribuant à enrichir le volume et la qualité des données disponibles, leur exploitation propose de nouvelles possibilités, incluant l'intégration, le par-

\section{Numérique et santé (11) \\ Intérêt des \\ dossiers de santé \\ électroniques \\ hospitaliers pour la \\ recherche clinique}

\section{Le projet européen}

EHR4CR

Danielle Dupont ${ }^{1 *}$, Ariel Beresniak ${ }^{1}$, Dipak Kalra ${ }^{2,3}$,

Pascal Coorevits ${ }^{3}$, Georges De Moor ${ }^{3}$

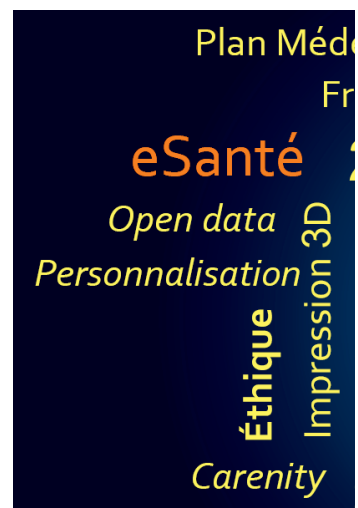

${ }^{1}$ Data Mining International, World trade center II, 29 Route de Pre-Bois, CH 1215, Geneve 15, Suisse.

${ }^{2}$ The European Institute for Health Records (EuroRec), De Pintelaan 185, Gand 9000, Belgique.

${ }^{3}$ Ghent university, department of public health, unit of medical informatics and statistics, De Pintelaan 185, Gand B9000, Belgique. info@datamining-international. tage et la mutualisation des données pour la recherche [6-8], pour l'aide à la décision thérapeutique, et pour l'optimisation des parcours de soins $[4,7]$.

com

*Danielle Dupont était chef de la direction scientifique chez Data Mining International S.A. jusqu'en mars 2018.

Le secteur de la recherche clinique est soumis à un nombre et une complexité de plus en plus soutenus des études cliniques. Au-delà des essais conventionnels visant à évaluer l'efficacité, l'innocuité et la qualité des innovations pharmaceutiques, les programmes de recherche clinique incluent de plus en plus des études pragmatiques de type «real world » visant à générer des données en situation réelle, ainsi que des études coût-efficacité permettant de comparer les coûts et l'efficacité d'une stratégie thérapeutique à ceux d'une situation de référence. Enfin, le secteur de la recherche clinique subit des enjeux importants, dont des délais significatifs pour l'exécution des études cliniques [9-11]. Ceux-ci ont pour conséquence négative de retarder l'introduction de thérapies innovantes au sein des systèmes de santé, et de contribuer ainsi à l'augmentation des coûts de recherche et développement.

Parmi les principaux enjeux rencontrés, citons le besoin d'évaluer plus précisément la faisabilité d'un protocole d'étude avant son lancement, la difficulté accrue de recruter des patients, en raison de la quantité et de la complexité des protocoles, ainsi que la lourdeur administra- 
tive liée à la collecte et au traitement des données cliniques [12]. Ces facteurs sont responsables de nombreux amendements aux protocoles, dont certains sont nécessaires pour gérer les imprévus inhérents au déroulement des essais. En effet, des difficultés souvent rencontrée est le décalage entre les études de faisabilité (réalisées en amont) et la réalité (en aval) [13]. Il est ainsi estimé que plus de $50 \%$ des protocoles nécessitent au moins un amendement, la moyenne étant de 2,3 amendements par protocole, et plus de 3,5 pour des études de phase $3[14,15]$. Chaque amendement compterait en moyenne 6,9 modifications au protocole initial ; les études de phase 3 en compteraient 8,5 , soit le plus grand nombre par amendement [10]. Le temps médian total requis pour la gestion d'un amendement est estimé à plus de 60 jours [10], et le coût moyen par amendement à 450000 dollars $[10,14,15]$. À l'évidence, ces aménagements entraînent d'importants délais et retards qui contribuent à la hausse significative des coûts de la recherche clinique. Les coûts de recherche et développement d'une nouvelle entité chimique ou biologique sont aujourd'hui estimés entre 142 millions et 1,75 milliard d'euros $[11,16]$. En améliorant la précision des analyses de faisabilité des protocoles d'études, en accélérant le ciblage et le recrutement des patients, et en automatisant le transfert et la gestion des données, de nombreuses causes de retards et des coûts importants pourraient être évités.

\section{Le projet Européen EHR4CR}

Bénéficiant d'un cofinancement de la Commission européenne et de la Fédération européenne des industries et associations pharmaceutiques (EFPIA) dans le cadre du programme IMI (innovative medicines initiative) ${ }^{1}$, le projet $\varepsilon H R 4 C R$ (electronic health records for clinical research) fût animé par un consortium public-privé entre 2011 et 2015 $[7,8,17]$. Les principaux objectifs du projet $\varepsilon H R 4 C R$ consistaient à développer et à tester une plateforme technologique pilote permettant de réutiliser les données cliniques contenues dans les DSE pour la recherche clinique et à concevoir un modèle économique (business model) permettant son exploitation évolutive et pérenne en Europe. Cette plateforme technologique innovante et conçue spécialement, est destinée à perfectionner les procédures actuelles de la recherche clinique, notamment: (1) en améliorant le plan expérimental et la précision des analyses de faisabilité des protocoles avant études; (2) en facilitant le choix et le recrutement des patients; et (3) en accélérant le transfert et le traitement des données cliniques ainsi que la notification et la déclaration des événements indésirables en cours d'études. En plus d'améliorer et d'accélérer l'exécution des essais cliniques, cette solution innovante promettait également d'attirer davantage d'investissements en recherche et développement en Europe, d'ouvrir de nouveaux axes de recherche, de générer de nouvelles sources de revenus pour les hôpitaux, et d'exploiter de nouvelles opportunités commerciales dans ce secteur à fort développement.

\footnotetext{
${ }^{1}$ http://www.imi.europa.eu/
}

\section{Identification des acteurs et enjeux en recherche clinique}

Afin de bien définir les différents acteurs impliqués, ainsi que les principaux enjeux en recherche clinique, le consortium EHR4CR a réalisé une enquête auprès d'un large panel d'intervenants experts en Europe [12]. L'objectif de cette enquête visait à préciser le rôle de tous les intervenants, leurs besoins spécifiques, ainsi que les opportunités économiques, les priorités et les facteurs de succès à considérer pour guider le développement d'un modèle qui soit pérenne en Europe. Les résultats de cette enquête européenne ont confirmé les défis actuels pour la planification et la réalisation d'études cliniques, de même que l'intérêt d'améliorer les analyses de faisabilité des protocoles, de mieux cibler et d'accélérer le recrutement des patients éligibles, d'optimiser le transfert des données, et de réduire les coûts.

\section{Méthodologies développées par le projet EHR4CR}

Plus de 34 partenaires académiques et 10 laboratoires pharmaceutiques ont collaboré au projet de recherche EHR4CR, déployant leurs expertises et meilleures pratiques afin de développer une plateforme technologique innovante permettant d'exploiter les données de DSE hospitaliers pour la recherche clinique en vue de proposer une solution digne de confiance, évolutive et durable.

Afin de s'assurer que les exigences et standards les plus élevés soient rencontrés en matière de conformité technologique, éthique, réglementaire, légale, et de respect de la confidentialité et de la protection des données personnelles $[7,8,17]$, des tests rigoureux furent réalisés avec une plateforme pilote, dans plus de 10 centres hospitaliers en Europe. Ces essais pilotes ont confirmé la qualité et la fiabilité des nombreuses fonctionnalités de la plateforme, ainsi que sa conformité et acceptabilité au plan éthique, chaque centre hospitalier ayant dû obtenir l'approbation préalable de son comité d'éthique. Le consortium a également conçu un programme d'accréditation pour les prestataires de services, incluant une certification formelle par tests de conformité des nouveaux produits et services offerts. Ainsi, la plateforme technologique a été testée de manière indépendante et rigoureuse par des experts de la sécurité afin de s'assurer que les promoteurs d'études ne puissent accéder à des informations exposant l'identité des individus ou à des caractéristiques de petits groupes de patients qui permettraient de les repérer. Seuls les noms agrégés des patients, dérivés des données des DSE hospitaliers pertinentes à un essai clinique proposé, peuvent être divulgués via la plateforme. 
Des méthodologies innovantes [18] furent également déployées afin d'optimiser l'adoption de la plateforme par les organisations de recherche, centres hospitaliers, et investigateurs cliniques, ainsi que pour en estimer sa valeur ajoutée, comparativement aux pratiques actuelles. La robustesse de l'ensemble des méthodologies utilisées dans le cadre du projet EHR4CR et leurs résultats ont ensuite permis de développer des propositions de valorisation adaptées, décrivant les bénéfices attendus pour chacun des intervenants concernés.

\section{Construction d'un modèle pérenne}

Afin que cette plateforme innovante puisse être implémentée de manière évolutive et durable, un modèle a été construit à l'échelle européenne, eu égard aux opportunités, besoins et intérêts de l'ensemble des acteurs impliqués en recherche clinique. L'agence de recherche Suisse Data Mining International S.A. ${ }^{2}$, spécialisée en recherche évaluative et stratégies de valorisation et de modélisation, a été mandatée par le consortium EHR4CR pour développer ce modèle. Pour cela, Data Mining International a constitué un groupe de travail multidisciplinaire, composé des principaux partenaires experts du projet. Ce groupe de travail, réuni une fois par trimestre, a collaboré à la conception des analyses d'affaires et enquêtes réalisées auprès des promoteurs d'études cliniques, centres hospitaliers, chercheurs, investigateurs, agences et prestataires en recherche clinique, professionnels de la santé, associations de patients, experts en technologies de l'information, etc. Sa mission consistait également à définir des chaînes de valeur multipartites et des propositions de valorisation uniques, adaptées aux besoins de chaque groupe d'intervenants ciblés, telles que révélés par l'enquête européenne [12]. Tout au long du projet, des ateliers annuels de consultation stratégique furent organisés auprès de nombreux responsables en recherche clinique pharmaceutique dans le but d'affiner les stratégies de modélisation et de valorisation.

Une étape préparatoire importante a visé à définir les spécificités de cet écosystème complexe, les plus importantes causes de retards, ainsi que les ressources et coûts évitables dans la planification et la réalisation d'essais cliniques. Ceci a permis de valider les fonctions clés que la plateforme devait fournir, ainsi que les domaines potentiels de son impact sur le flux de travail et les coûts des essais cliniques. Les résultats de ces expertises ont également permis de développer des méthodologies de valorisation appropriées pour tous les acteurs de cet écosystème.

Des propositions de valorisation furent ensuite élaborées et adaptées tenant compte des besoins non comblés révélés par l'enquête européenne [12]. Centrales au modèle, et telles que résumées dans le Tableau l, ces propositions ont permis de décrire les bénéfices spécifiques que cette plateforme innovante et ses nouveaux services attenants allaient pouvoir générer pour l'ensemble des parties prenantes (les hôpitaux, organisations de recherche, investigateurs cliniques, systèmes de santé, patients, prestataires de services, promoteurs d'études cliniques, industries de technologies de l'informa-

${ }^{2}$ www.datamining-international.com tion et autres intervenants) [19]. Ces propositions de valorisation adaptées étant cruciales pour promouvoir les bénéfices attendus auprès de chaque groupe d'intervenants, elles permettent d'optimiser la recevabilité, le succès, et donc la valeur ajoutée des solutions proposées.

Concernant les attentes spécifiquement exprimées par les promoteurs d'études cliniques en Europe, Data Mining International a également réalisé des simulations coût-bénéfices [20] et d'incidence budgétaire [21], lesquelles ont permis d'estimer la valeur ajoutée ainsi que les économies potentielles que cette nouvelle plateforme technologique était en mesure de réaliser en Europe. Ces études ayant confirmé que les solutions apportées s'avéraient plus efficaces et plus économiques que les pratiques actuelles, ces évidences ont contribué à consolider les propositions de valorisation destinées aux promoteurs d'études cliniques, en vue d'assister leurs décisions d'investissement [19].

Data Mining International a adapté au modèle une méthodologie d'évaluation par simulations quantitatives qui a établi que l'exploitation des données de DSE hospitaliers pour la recherche clinique était susceptible de constituer un axe de développement majeur pour les prestataires de ces nouveaux services en Europe [19]. La prise en compte des hypothèses financières et des incertitudes inhérentes à l'investigation de stratégies innovantes étant essentielle, des analyses de sensibilité probabilistes de type Monte-Carlo ont été utilisées, confirmant la robustesse des résultats. Ces analyses concluantes ont permis d'assister les décisions d'investissement des prestataires de ces nouveaux services fournis par cette plateforme innovante.

Afin de stimuler l'innovation pour la réutilisation des informations numériques pour la recherche, EHR4CR a été à l'initiative de la création de l'I HD ${ }^{3}$ (european institute for innovation through health data), une organisation européenne à but non lucratif dont le rôle est de réguler, développer et promouvoir les axes de collaborations dans ce domaine auprès de l'ensemble des intervenants du secteur de la recherche. Malgré l'attention particulière et les soins apportés à la conception de la plateforme EHR4CR pour protéger les données personnelles confidentielles, il a été reconnu qu'un travail à plus long terme était nécessaire en Europe pour développer et promouvoir les bonnes pratiques auprès de toutes les communautés de recherche clinique en ce qui concerne la protection de la vie privée et la collecte de données de santé électroniques normalisées de haute qualité. La création d'un nouvel organisme dédié à cette mission, compétent au

\footnotetext{
${ }^{3}$ http://www.i-hd.eu/
} 


\begin{tabular}{|c|c|}
\hline Acteurs & Bénéfices d'utilisation de la plateforme technologique \\
\hline $\begin{array}{l}\text { Hôpitaux, centres de recherche } \\
\text { et investigateurs cliniques }\end{array}$ & $\begin{array}{l}\text { - Participation accrue et efficiente à des programmes de recherche } \\
\text { - Amélioration de la qualité des données et des connaissances } \\
\text { - Facilitation des processus et augmentation des revenus en recherche }\end{array}$ \\
\hline Promoteurs d'études & $\begin{array}{l}\text { - Efficience accrue pour la planification et la réalisation des études cliniques } \\
\text { - Réduction du temps et des coûts pour la réalisation d'études } \\
\text { - Réalisation d'une valeur ajoutée substantielle }\end{array}$ \\
\hline Patients & $\begin{array}{l}\text { - Participation à des études cliniques axées sur les résultats de santé } \\
\text { - Contribution à l'amélioration des connaissances en gestion optimale de la santé } \\
\text { - Accès plus rapide à des thérapies innovantes }\end{array}$ \\
\hline Prestataires de services & $\begin{array}{l}\text { - Exploitation profitable de nouveaux services de pointe } \\
\text { - Amélioration de l'efficience et développement de nouvelles compétences } \\
\text { - Expansion et croissance des activités commerciales }\end{array}$ \\
\hline $\begin{array}{l}\text { Industrie des technologies de } \\
\text { l'information }\end{array}$ & $\begin{array}{l}\text { - Développement d’outils technologiques de pointe pour la recherche } \\
\text { - Exploitation de nouveaux créneaux commerciaux } \\
\text { - Croissance du marché }\end{array}$ \\
\hline $\begin{array}{l}\text { Agences réglementaires, } \\
\text { professionnels et systèmes de santé }\end{array}$ & $\begin{array}{l}\text { - Évidences de l'efficacité et d'innocuité cliniques générées plus rapidement } \\
\text { - Notification plus rapide des événements indésirables } \\
\text { - Accès plus rapide à des évidences probantes pour assister les décisions }\end{array}$ \\
\hline
\end{tabular}

Tableau I. Bénéfices des solutions EHR4CR/InSite (d'après [19]).

niveau européen, a été considérée comme la meilleure façon de diffuser ces bonnes pratiques. De par sa mission de gouvernance, ce nouvel institut devrait guider l'établissement de meilleures pratiques, faciliter l'exploitation pour la recherche des données numériques cliniques générées par le fort développement des technologies d'information en santé, et contribuer à accroître les opportunités de recherche et de développement dans ce secteur émergeant [22].

\section{Une solution technologique éprouvée en réponse aux défis actuels}

Les acteurs de la recherche clinique assistent actuellement à une demande croissante du nombre et de la complexité des études cliniques à réaliser, incluant les études conventionnelles, les études pragmatiques de type «real world», et les études coût-efficacité. Le projet de plateforme développé par EHR4CR rend désormais possible la réutilisation et l'exploitation des données numériques contenues dans les DSE hospitaliers pour la recherche, proposant ainsi des solutions innovantes aux enjeux identifiés. Parce qu'elle permet d'améliorer le plan expérimental des études, de mieux cibler et de recruter les patients plus rapidement, et de faciliter l'échange des données électroniques en cours d'étude, la plateforme développée par le projet de recherche $\varepsilon H R 4 C R^{4}$, représente une réelle avancée de modernisation des processus actuels en recherche clinique. Tel qu'illustré dans les Figures 1-3, et comme le suggère son libellé «InSite », la plateforme

${ }^{4}$ Depuis commercialisée sous le label InSite : https://www.insiteplatform.com/ exécute à distance les analyses sur les données de DSE hospitaliers (remote analytics) : aucune donnée de patient ne quitte l'hôpital, les calculs étant exécutés dans les sites hospitaliers. Seuls les résultats d'analyses sont envoyés aux organismes de recherche en charge des études cliniques, et ce, de manière sécurisée et digne de confiance.

En plus d'améliorer les pratiques, cette plateforme permet d'ouvrir de nouveaux axes de recherche, de générer des revenus de recherche additionnels pour les hôpitaux et centres de recherche, ainsi que d'exploiter de nouvelles opportunités commerciales. Le programme d'évaluation, conçu spécialement dans le cadre du projet $\varepsilon H R 4 C R$, a non seulement confirmé que cette plateforme entraîne une réduction des coûts d'exécution des essais cliniques [21], mais a aussi établi qu'elle génère une véritable valeur ajoutée pour les promoteurs d'études cliniques [20], contribuant à accélérer le développement et la mise sur le marché des innovations thérapeutiques en Europe et dans le monde.

Une prochaine étape consistera à évaluer et à comparer la performance de ce type de plateformes auprès de l'ensemble des intervenants visés dans un contexte d'utilisation en situation réelle [20]. Une méthodologie d'évaluation multicritères est en cours de développement pour définir des indicateurs pertinents (critères de performance, de succès, de temps, de coûts, de satisfaction 


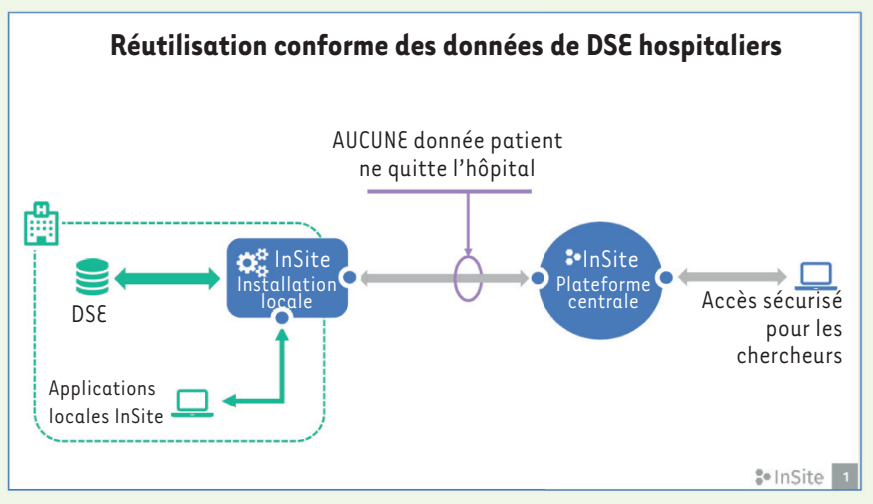

Figure 1. Analyses réalisées à distance par la plateforme InSite. DSE : dossiers de santé électroniques.

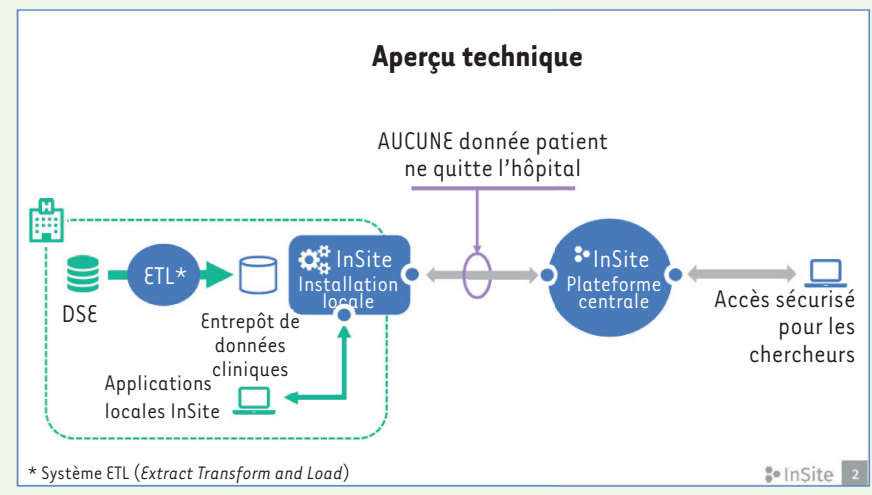

Figure 2. Intégration, transformations appliquées et livraison de résultats agrégés par InSite. DSE : dossiers de santé électroniques; ETL : extract transfom and load.

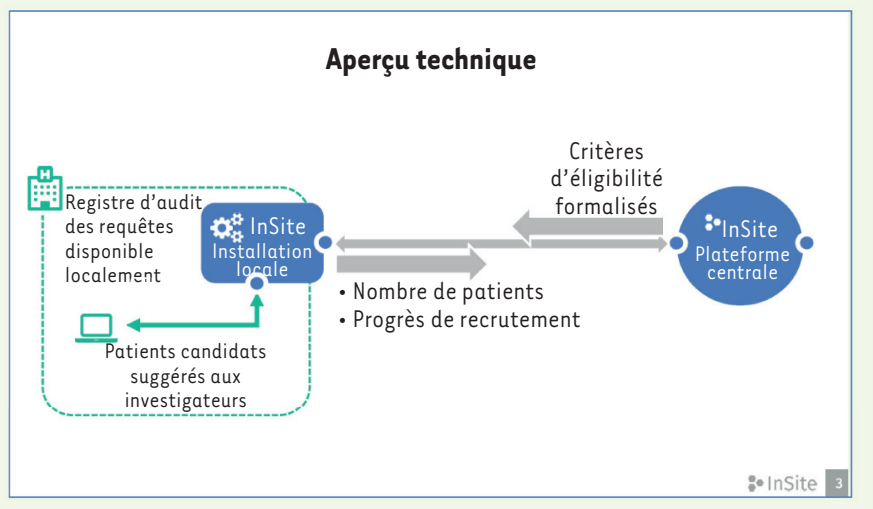

Figure 3. Réutilisation des données de DSE hospitaliers en recherche clinique par InSite. DSE : dossiers de santé électroniques.

des acteurs, etc.). Pour cela, les performances et les coûts seront évalués en termes d'efficacité au niveau d'un ou plusieurs hôpitaux majeurs en Europe.

\section{Conclusion}

L'exploitation sécurisée des données cliniques numérisées contenues dans les DSE hospitaliers permet non seulement d'analyser les informations disponibles pour le bénéfice des patients et de la société, mais contribue aussi à élargir les domaines de recherche en étroite collaboration avec les hôpitaux et autres organisations de recherche. Ces progrès devraient permettre de concevoir de nouveaux modèles de soins de santé intégrés axés sur l'optimisation des résultats de santé [23-25]. Grâce au développement de systèmes interopérables de plus en plus performants, l'exploitation des bases de données cliniques numérisées pour la recherche clinique est aujourd'hui opérationnelle $[4,7,8,17]$. $\varepsilon$ n améliorant l'efficience des processus actuels, les plateformes d'exploitation innovantes telles que celle développée dans le cadre du projet EHR4CR permettent désormais de mener un grand nombre d'études cliniques et observationnelles complexes plus efficacement et plus rapidement, de réduire les coûts de développement, et d'accélérer l'avènement de nouvelles thérapies pour répondre à des besoins médicaux non satisfaits. L'évaluation coûts-bénéfices [20] et l'analyse d'incidence budgétaire [21] réalisées dans le cadre du projet EHR4CR ont également permis d'établir l'importance de la valeur ajoutée, de même que les économies potentielles pouvant être générées par cette plateforme dédiée auprès des promoteurs d'études et autres organisations de recherche en Europe. Ce modèle spécifique a aussi démontré l'intérêt et le potentiel de rentabilité économique pour les prestataires de services, en vue du déploiement pérenne de ces solutions sur le marché européen [19]. Ces analyses étant fondées sur des modèles de simulation, il s'avère désormais nécessaire de concevoir un programme d'évaluation de ce type de plateformes en contexte réel afin de consolider les propositions de valorisation existantes auprès de l'ensemble des acteurs de la recherche clinique. Ces analyses ultérieures contribueront au déploiement évolutif de ces innovations technologiques en Europe et audelà, ainsi qu'à l'amélioration continue des pratiques. $\diamond$

\section{SUMMARY}

Value of hospital electronic health records for clinical research: contribution of the European project

\section{हHR4CR}

Electronic health records in hospitals contribute to improving the quality of care by enabling better management of clinical information. The databases thus constituted facilitate the exchange of health information with healthcare providers and optimize multidisciplinary coordination 
for better therapeutic results. The EHR4CR (Electronic Health Records for Clinical Research) European project has developed an innovative pilot platform enabling the reuse of this digital information for clinical research. By enhancing and speeding up clinical research procedures, this innovative approach makes it possible to conduct clinical trials more efficiently, faster, and more economically. $\diamond$

\section{LIENS D'INTÉRÊT}

Les auteurs déclarent n'avoir aucun lien d'intérêt concernant les données publiées dans cet article.

\section{RÉFÉRENCES}

1. Geissbuhler A, Safran C, Buchan I, et al. Trustworthy reuse of health data: A transnational perspective. Int J Med Inform 2013 ; 82 : 1-9.

2. Gunter TD, Terry NP. The emergence of national electronic health record architectures in the United States and Australia: models, costs, and questions. J Med Internet Res $2005 ; 7(1)$ : e3.

3. Higgins JK. Electronic Health Records: Big Business, and Getting Bigger. TechNewsWorld Best of ECT News. 2010 (May 14). http://www.crmbuyer.com/story/69994.html,

4. Jensen PB, Jensen LJ, Brunak S. Mining electronic health records: towards better research applications and clinical care. Nat Rev Genet 2012 ; 13(6) :395-405

5. Bateman S. Médecine personnalisée, un concept flou, des pratiques diversifiées. Med/Sci (Paris) 2014 ; 30 (hors série ${ }^{\circ} 2$ ) : 8-13.

6. Boudry C. Biologie/Médecine 2.0 : état des lieux. Med/Sci (Paris) $2012 ; 28: 653-8$.

7. Coorevits $P$, Sundgren $M$, Klein G0, et al. Electronic health records: new opportunities for clinical research. J Intern Med $2013 ; 274(6)$ : 547-60.

8. De Moor G, Kalra D, Sundgren M, et al. Opportunities for clinical research in European hospitals: the EHR4CR platform. Health management $2014 ; 14: 59-60$.

9. Tufts. Analysis and insight into critical drug development issues. Tufts University Center for the Study of Drug Development (CSDD). Impact Report 2013 ; 15(1) : 1-4.

10. Tufts. Rising protocol complexity, execution burden varies widely by phase and therapeutic areas (TA). Tufts University Center for the Study of Drug Development (CSDD) Report 2010 ; 12 (3).

11. Sertkaya A, Birkenbach A, Berlind A, Eyraud J. Examination of Clinical Trial Costs and Barriers for Drug Development. ASPE 2014 July 25. http://aspe.hhs.gov/sp/reports/2014/ClinicalTrials/rpt_erg.pdf.

12. Kalra D, Schmidt A, Potts HWW, et al. Case report from the EHR4CR project - A european survey on electronic health records systems for clinical research. iHealth Connections $2011 ; 1$ (2) : 108-13.
13. Micallef J. Table ronde 3 : Comment améliorer la compétitivité de la France en recherche clinique. Med/Sci (Paris) $2014 ; 30: 28-32$.

14. Getz K, Wenger J. Assessing the impact of protocol design change on clinical trial performance. Am J Ther $2008 ; 15: 449-56$.

15. Alsumidaie M. Aggregated EMR: Mitigating trial risk through quality by design protocols. Applied Clinical Trials online 2014 ; August. https:// www.linkedin.com/pulse/20140828131707-55450526-aggregated-emrmitigating-trial-risk-through-quality-by-design-protocols.

16. Mestre-Ferrandiz J, Sussex J, Towse A. The R\&D cost of a new medicine. Office of Health Economics 2012.

17. De Moor G, Sundgren M, Kalra D, et al. Using electronic health records for clinical research: the case of the EHR4CR project. J Biomed Inform 2014 ; $53: 162-73$.

18. Osterwalder A, Pigneur Y. Business Model Generation. PEARSON EDUCATION 2009, 288p.

19. Dupont D, Beresniak A, Sundgren M, et al. Business analysis for a sustainable, multi-stakeholder ecosystem forleveraging the Electronic Health Records for Clinical Research (EHR4CR) platform in Europe. Int J Med Inform 2017 ; 97 : 341-52.

20. Beresniak A, Schmidt A, Proeve J, et al. Cost-benefit assessment of using electronic health records data for clinical research versus current practices: Contribution of the Electronic Health Records for Clinical Research (EHR4CR) European Project. Contemp Clin Trials 2016 ; 46 : 85-91.

21. Dupont D, Beresniak A, Schmidt A, et al. Assessing the Financial Impact of Reusing Electronic Health Records Data for Clinical Research: Results from the EHR4CR European Project. J Health Med Informat 2016 ; 7(3) : 235.

22. Kalra D, Stroetmann V, Sundgren $M$, et al. The European Institute for Innovation through Health Data. Learn Health Sys, 2017 ; 1: p. 1-8.

23. Salimi T, Lehner JP, Epstein RS. A framework for pharmaceutical value-based innovations. J Comp Eff Res 2012 : 1(Suppl. 1) : 3-7.

24. Lehner JP, Epstein RS, Salimi T. Integrating new approaches for clinical development: translational research and relative effectiveness. J Comp Eff Res 2012 ; 1(1 Suppl) : 15-21.

25. Epstein R, Sidorov J, Lehner JP, Salimi T. Integrating scientific and real-world evidence within and beyond the drug development process. J Comp Eff Res 2012 ; l(1 Suppl) : 9-13.

TIRÉS À PART

D. Dupont

u'est-ce que la guérison? Des réponses, il y en a. De toutes sortes et de tout temps. Chacun y va de son savoir, religieux, scientifique, médical... Et de quoi est-on supposé guérir ? D’un symptôme, d'une douleur, d'une maladie, d'une répétition mortifère, d'un destin mélancolique? Pour la psychanalyse, la guérison s'insère dans un système imaginaire et a, comme point de mire, un idéal. «La guérison, c’est une demande... » précise Lacan. Les auteurs nous invitent ici à découvrir, au-delà du semblant et à partir de la clinique, les liens entre guérison et vérité du sujet.

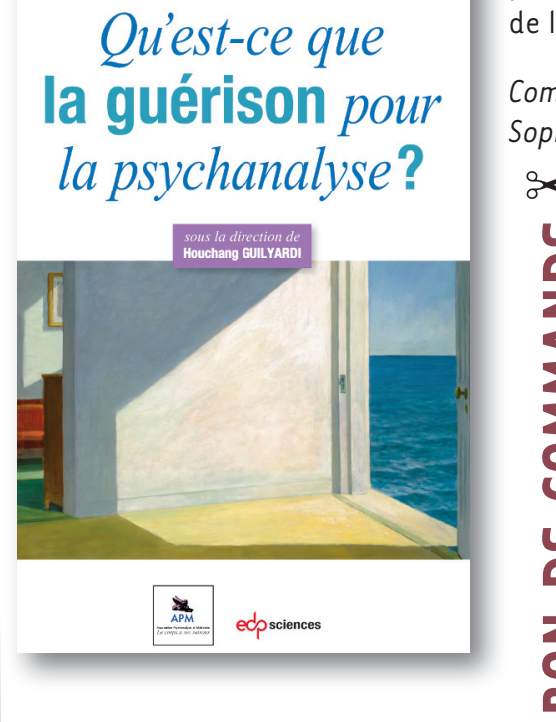

Comité éditorial de l'Association Psychanalyse et Médecine (APM) : Martine Dombrosky, Sophie Dunoyer de Segonzac, Houchang Guilyardi, Josette Olier, Betty Testud

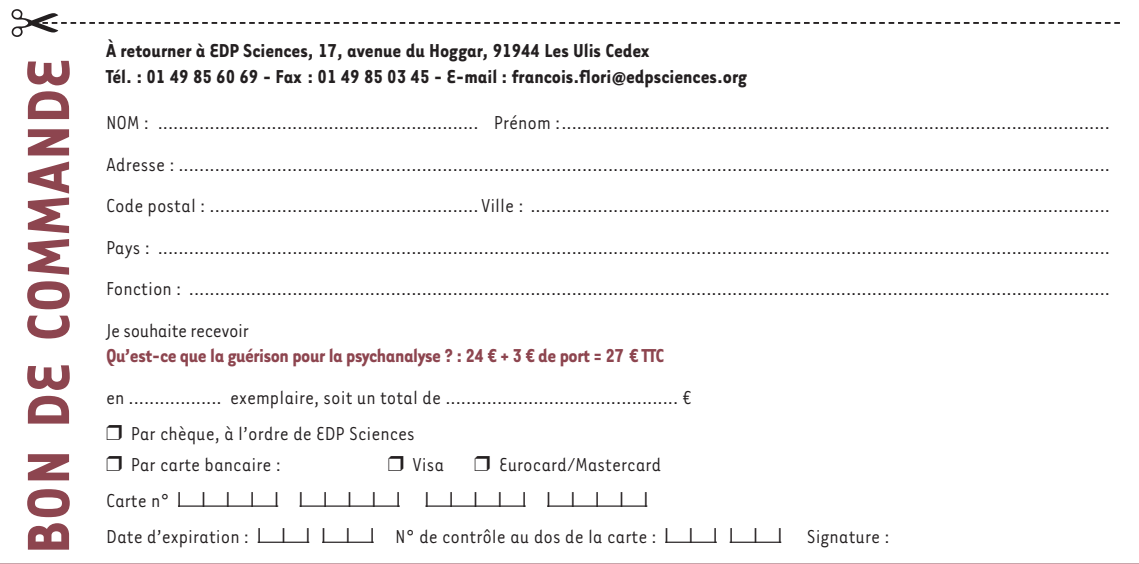

